\title{
Cinemática inversa de un robot redundante tipo scorbot - ER Vplus: influencia de índices de desempeño
}

\section{Inverse kinematics of a redundant robot type scorbot - ER Vplus: influence of performance indices}

\author{
Luz Adriana Mejía-Calderón \\ Ph.D. Ingeniería y Producción Industrial \\ Universidad Tecnológica de Pereira \\ Pereira, Colombia \\ adriamec@utp.edu.co
}

\author{
Alexander González-Echeverry \\ Ingeniero Mecánico \\ Universidad Tecnológica de Pereira \\ Pereira, Colombia \\ alexander.echeverri@busscar.com.co
}

\author{
Héctor Fabio Quintero-Riaza \\ Ph.D. Ingeniería \\ Universidad Tecnológica de Pereira \\ Pereira, Colombia \\ hquinte@utp.edu.co
}

\begin{abstract}
Resumen- En este trabajo se realiza la cinemática inversa de un robot redundante tipo Scorbot-ER VPlus, al que se le ha añadido un grado de libertad adicional, consistente en una base deslizante. Para la solución del problema cinemático inverso se aplica un proceso iterativo de evaluación del desempeño del manipulador en diferentes configuraciones. Se selecciona una coordenada de nodo como variable en el proceso y fijando su valor, se determinan las demás coordenadas a partir del modelo cinemático inverso del manipulador original. Para esta configuración se evalúan diversos índices de desempeño aplicados a sistemas robóticos y se analiza el costo computacional de su inclusión en la solución del problema cinemático inverso. Posteriormente se varía la coordenada seleccionada y se repite el proceso. De esta manera es posible evaluar para qué índices de desempeño se logran menores tiempos de resolución, buscando su aplicación e inclusión dentro del control cinemático del robot redundante.
\end{abstract}

Palabras clave- Índice de desempeño, manipulabilidad, matriz jacobiana, redundancia cinemática, robot.

Abstract- In this paper the inverse kinematics of a redundant Scorbot-ER VPlus type robot with an additional degree of freedom, represented by a sliding platform, is performed. For the solution of the inverse kinematic problem it is developed an iterative process that evaluates the performance of the manipulator in different configurations. A joint coordinate is selected as a variable in the iterative process and setting its value, the other coordinates are determined from the inverse kinematic model of the original manipulator. For this configuration different performance indices applied to robotic systems are evaluated and the computational cost of their inclusion in the solution of the inverse kinematic problem is analyzed. Subsequently the selected coordinate is varied and the process is repeated. In this way it is possible to as- sess the performance indices leading to lower resolution times, seeking their application and inclusion in the kinematic control of redundant robot.

Keywords- Performance indexes, manipulability, Jacobian matrix, kinematic redundancy, robot.

\section{INTRODUCCIÓN}

En el campo de la robótica, los sistemas redundantes, es decir, aquellos que poseen más grados de libertad de los necesarios para desarrollar una tarea, son utilizados como métodos de flexibilización y reconfiguración del sistema, pues permiten aumentar el espacio de trabajo del robot y posibilitan también la eliminación de algunas configuraciones singulares al activar esos grados de libertad adicionales [1]-[3]. La adición de tales características si bien beneficia la movilidad del sistema desde el punto de vista funcional, presenta su inconveniencia en la dificultad de resolver el problema cinemático inverso. Para robots serie, la cinemática inversa, que precisa de encontrar los valores de las coordenadas de nodo en función de la posición y orientación del elemento terminal del manipulador es un problema no trivial; al ser un sistema no lineal, presenta varias soluciones para una posición y orientación específica del elemento terminal [4]-[6]. En la mayoría de los casos, las restricciones en los pares cinemáticos y algunas consideraciones de tipo energético limitan ese abanico de soluciones a unos pocos conjuntos 
de coordenadas nodales, siendo importante optimizar el algoritmo implementado con el fin de reducir el costo computacional del proceso. Sin embargo, para robots redundantes, los conjuntos de coordenadas nodales que resuelven el problema cinemático inverso son infinitos, por lo que es necesario añadir criterios de selección adicionales [1]. En este sentido, se plantea la evaluación del desempeño del manipulador como criterio de selección del mejor conjunto de coordenadas de nodo. Dado que existen diversos índices de desempeño aplicados a sistemas robóticos, en este trabajo, se evalúan cuatro diferentes índices de desempeño y se analiza el costo computacional de su inclusión en la solución del problema cinemático inverso para un robot tipo Scorbot al que se le ha adicionado una base deslizante.

En la Sección 2 se presenta la elaboración del modelo cinemático inverso del manipulador Scorbot VPlus en base deslizante. En la Sección 3 se presentan algunos índices de desempeño utilizados en sistemas robóticos y se seleccionan los índices que serán evaluados. Luego se resuelve el problema cinemático inverso con los índices de desempeño y los resultados obtenidos son presentados en la Sección 4.

\section{MODELO CINEMÁTICO INVERSO DEL MANIPULADOR SCORBOT-ER VPLUS EN BASE DESLIZANTE}

El robot Scorbot ER-VPlus que tiene el Laboratorio de Manufactura de la Facultad de Mecánica de la Universidad Tecnológica de Pereira se ha incorporado dentro una celda de manufactura flexible que cuenta además con un sistema de visión(a), un torno(b) y una mesa revolver(c), tal como aparece en la Fig 1.A. Para realizar las tareas de manutención de piezas para el torneado y el sistema de verificación, al robot se le ha adicionado un grado de libertad más, consistente en una base deslizante, que le permite desplazarse sobre toda la mesa de trabajo de la celda, Fig 1.B.

Si bien, es evidente el aporte en flexibilidad tras la adición del grado de libertad, ahora cuenta con 6 grados de libertad (G.D.L), el nuevo sistema requiere un nuevo algoritmo de resolución de la cinemática directa e inversa. En el caso de la cinemática directa, es suficiente con premultiplicar la matriz de transformación de la base deslizante añadida, a partir del nuevo parámetro de Denavit-Hartenberg Modificado (DHM) a la matriz de transformación total original [7]. En la Fig. 2 se presenta la notación de los sistemas de referencia locales a partir de la notación DHM, teniendo en cuenta la posición inicial del robot (HOME), en cuyo caso las coordenadas de nodo $\theta_{2}, \theta_{3}$ y $\theta_{4}$ presentan valores iniciales (offsets), como se observa en la figura. Adicional a la consideración de valor inicial, una característica importante del manipulador Scorbot ER V-Plus es que el movimiento de algunas coordenadas nodales está asociado con los movimientos de las coordenadas inmediatamente anteriores, en procura de mantener constante la orientación del elemento terminal. Si por ejemplo, se gira la coordenada $\theta_{2}$ un ángulo de $20^{\circ}$ sin modificación de $\theta_{3}$, el sistema actúa de forma tal que modifica esta última coordenada en $-20^{\circ}$, para conservar el valor de $96^{\circ}$ como ángulo relativo entre los eslabones 3 y 4 (para la posición indicada en la figura). Los parámetros de DHM que definen la configuración del manipulador se presentan en la Tabla I.

Fig. 1. CELDA DE MANUFACTURA CON ROBOT SCORBOT

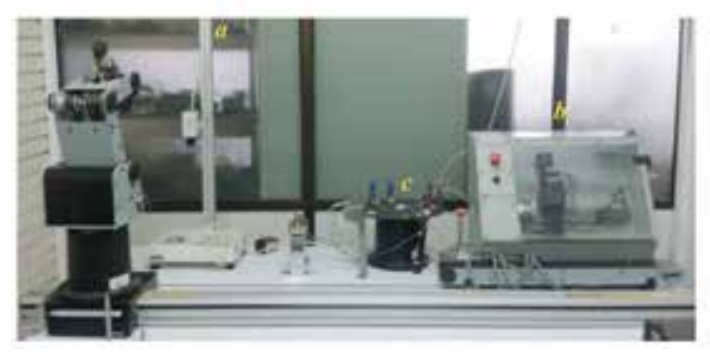

A)

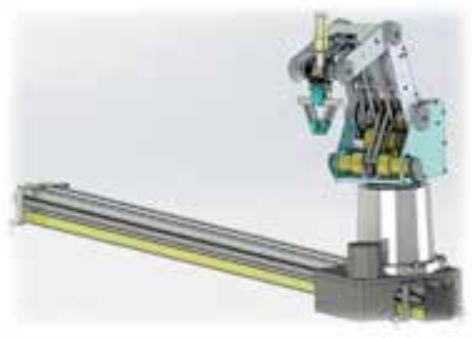

B)

Fuente: los autores. 
TABLA I

PARÁMETROS DHM MEDIDOS DESDE EL HOME PARA EL ROBOT SCORBOT-ER VPLUS

\begin{tabular}{|c|c|c|c|c|}
\hline & $\alpha$ & $\alpha$ & $\theta$ & $d$ \\
\hline $0-1$ & $\pi / 2$ & 0 & 0 & $d_{0}$ \\
\hline $1-2$ & $-\pi / 2$ & 0 & $\theta_{1}$ & $l_{1}$ \\
\hline $2-3$ & $\pi / 2$ & $a$ & $\theta_{2}+3 \pi / 5$ & 0 \\
\hline $3-4$ & 0 & $l_{2}$ & $\theta_{3}-\theta_{2}-8 \pi / 15$ & 0 \\
\hline $4-5$ & 0 & $l_{3}$ & $\theta_{4}-\theta_{3}+11 \pi / 60$ & 0 \\
\hline $5-6$ & $\pi / 2$ & 0 & $\pi / 2+\theta_{5}$ & $l_{4}$ \\
\hline
\end{tabular}

Fuente: los autores.

Fig. 2. PARÁMETROS DE DENAVIT-HARTENBERG MODIFICADOS CON OFFSETS

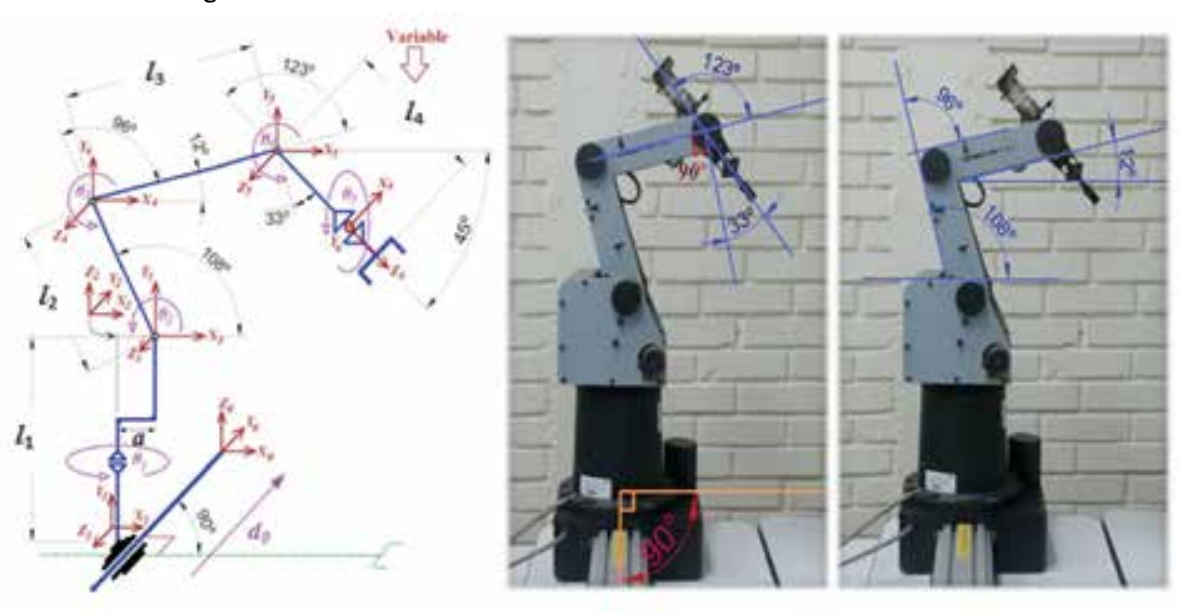

Fuente: los autores.

Una metodología usual para obtener la cinemática inversa en robots serie, es el denominado desacople cinemático [7], en donde se obtienen de forma independiente los primeros G.D.L. para el posicionamiento de la muñeca y los siguientes G.D.L. para la orientación, teniéndose como requisito que los 3 últimos G.D.L se intersecten en el mismo punto. El robot Scorbot ER V-Plus original, sin la base deslizante, posee 3 grados de libertad G.D.L. para el posicionamiento de la muñeca y solo 2 para la orientación, por lo que la orientación total del elemento terminal no está definida por los últimos grados de libertad, como ocurre en otros manipuladores (6 G.D.L.), sino que la orientación está definida también por la coordenada inicial $\theta_{I}$. La dirección del eje $Z$ del elemento terminal y la posición de la muñeca del manipulador (sistema de referencia 5), deben estar contenidas en el plano del brazo robótico, que es el plano vertical formado por el eje de la junta de $\theta_{1}$ y la posición de la muñeca, Fig 3. Por este motivo, el desacople cinemático si bien puede ser usado presenta restricciones con respecto a la orientación de $Z_{6}$.

En la cinemática inversa, el objetivo es encontrar las coordenadas nodales necesarias para alcanzar una posición y orientación determinadas del elemento terminal. Estos requisitos se integran en la matriz de transformación homogénea total, que incorpora la orientación (3 primeras columnas) y la posición (columna 4) del elemento terminal como se presenta en (1),

$$
{ }^{0} T_{6}=\left[\begin{array}{cccc} 
& & \}_{{ }_{k 6}}^{r} & \}_{0^{0}}^{r} \\
n_{x} & o_{x} & a_{x} & r_{x} \\
n_{y} & o_{y} & a_{y} & r_{y} \\
n_{z} & O_{z} & a_{z} & r_{z} \\
0 & 0 & 0 & 1
\end{array}\right]
$$


De la Fig. 3 se observa que la coordenada de nodo $\theta_{1}$ se puede obtener directamente del eje unitario de $Z_{6}$, cuyo valor se obtiene de la columna 3 de la matriz de transformación homogénea del manipulador cuando el manipulador es fijo (sin la base deslizante). Aún en la configuración de base fija, cuando la orientación del eje $Z_{6}$ es $(0$, $0, \pm 1)$, es decir, para $a_{x}$ y $a_{y}$ nulos, la coordenada de nodo deberá obtenerse a partir de la posición de la muñeca del manipulador, método conocido como desacople cinemático. Si se conoce la posición del elemento terminal, y sabiendo que los dos últimos sistemas de referencia locales son coincidentes, dicha posición se obtiene como,

$$
\vec{r}_{m}={ }^{0} \vec{r}_{6}-l_{4}{ }^{0} \vec{k}_{6}
$$

La longitud $l_{4}$ varía de acuerdo con el espesor de la pieza por manipular. Se puede observar en la Fig. 4-a que la distancia $l_{4}$ desde el eje de giro del elemento terminal (gripper) hasta el punto terminal es variable, por lo que debe introducirse la corrección del parámetro, valor no contemplado en el modelo cinemático original del manipulador.

Con los valores geométricos conocidos para el gripper, Fig. 5, se obtiene el valor de corrección de la longitud $l_{4}$. Considerando $x$ como el espesor de la pieza por sujetar se tiene que,

$$
l_{4}=89,55+\sqrt{2500-\left(20,84-\frac{x}{2}\right)^{2}}
$$

Fig. 3. ORIENTACIÓN DEL ELEMENTO TERMINAL CON RESPECTO A LA CINTURA DEL ROBOT

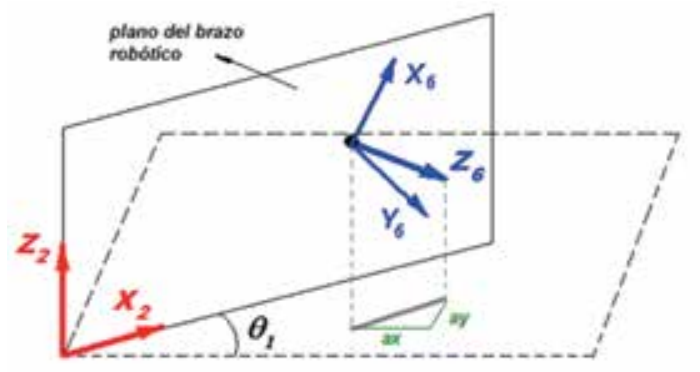

Fuente: los autores.

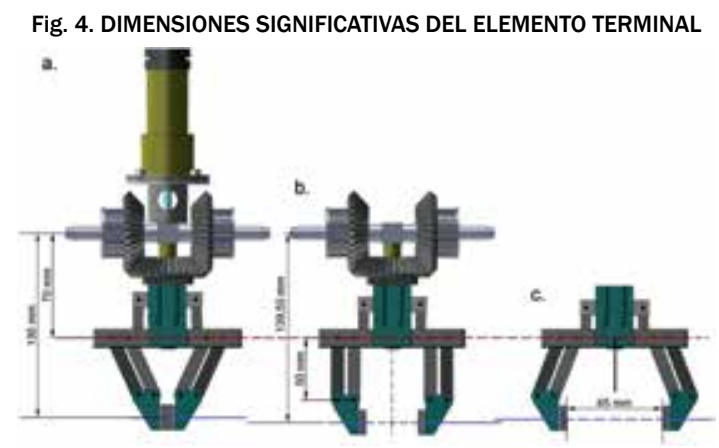

Fuente: los autores.

Fig. 5. DIMENSIONES DEL ELEMENTO TERMINAL

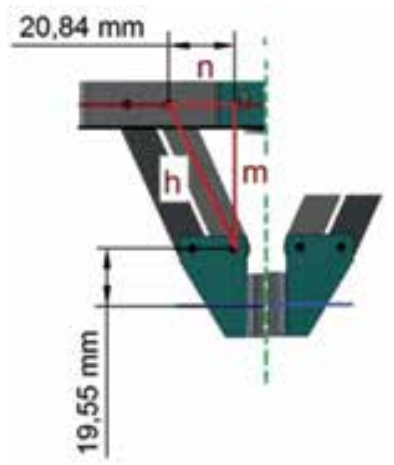

Fuente: los autores. 
Ahora bien, al incluir la base deslizante, el posicionamiento de la muñeca se obtiene a través de 4 G.D.L, uno más de los necesarios para posicionar un elemento en el espacio tridimensional. La coordenada de giro de la cintura del manipulador queda determinada como,

$$
\theta_{1}=a \tan \left(\frac{-a_{y} \cdot l_{4}+r_{y}-d_{1}}{-a_{x} \cdot l_{4}+r_{x}}\right)
$$

Siendo $\mathrm{d}_{1}$ la coordenada de la base deslizante. Las variables $a_{x}, a_{y}$ y $a_{z}$ son los valores asociados al vector unitario del eje $Z$ del elemento terminal (orientación del elemento terminal), y $r_{x}$ y $r_{y}$ son las posiciones $x$ e $y$ del elemento terminal de acuerdo con la matriz de transformación de la expresión (1).

Al aplicar la inversa de las matrices de transformación homogéneas ${ }^{i} T_{j}$ entre eslabones, tal como se presenta en las expresiones en (5), es posible determinar un sistema de ecuaciones algebraicas para las coordenadas de nodo $\theta_{2}$ y $\theta_{3}$,

$$
\begin{aligned}
& \left({ }^{0} T_{1}\right){ }^{-10} T_{5}={ }^{1} T_{2}{ }^{2} T_{3}{ }^{3} T_{4}{ }^{4} T_{5} \\
& \left({ }^{1} T_{2}\right)^{-1}\left({ }^{0} T_{1}\right)^{-10} T_{5}={ }^{2} T_{3}{ }^{3} T_{4}{ }^{4} T_{5} \\
& \left({ }^{2} T_{3}\right)^{-1}\left({ }^{1} T_{2}\right){ }^{-1}\left({ }^{0} T_{1}\right){ }^{-10} T_{5}={ }^{3} T_{4}{ }^{4} T_{5}
\end{aligned}
$$

Para lo cual se dará

$$
\begin{gathered}
\theta_{2}=\tan ^{-1}\left(\frac{Z_{m}-l_{1}}{\sqrt{X_{m}^{2}+\left(X_{m}+d\right)^{2}-a}}\right) \\
-\tan ^{-1}\left(\frac{l_{3} \sin \theta_{3}}{l_{3} \cos \theta_{3}+l_{2}}\right)-\frac{3 \pi}{5}- \\
\theta_{3}=\tan ^{-1}\left[\frac{\sqrt{1-\left(\frac{U}{2 \cdot l_{2} l_{3}}\right)^{2}}}{\frac{U}{2 \cdot l_{2} l_{3}}}\right]+\theta_{2}+\frac{8 \pi}{15}
\end{gathered}
$$

$$
\text { donde } \quad U=\left(Z_{m}-l_{1}\right)^{2}+X_{m}^{2}+\left(Y_{m}+d\right)^{2}+a^{2}
$$
$-2 a \sqrt{X_{m}^{2}+\left(Y_{m}+d\right)^{2}}-l_{3}^{2}-l_{2}^{2}$ para $X_{m}=r_{x}-a_{x} l_{4}$, $Y_{m}=r_{y}-a_{y} l_{4}$ y $Z_{m}=r_{2}-a_{z} l_{4}$. Con $a_{z}$ y $r_{z}$ como variables de orientación y posición del elemento terminal, según la expresión (1)

Las variables $\theta_{4}$ y $\theta_{5}$ se obtienen a partir de las matrices de rotación, tal que,

$$
{ }^{4} R_{6}={ }^{4} R_{5} \cdot{ }^{5} R_{6}=\left({ }^{0} R_{3}\right)-1 \cdot{ }^{0} R_{6}
$$

donde ${ }^{i} R_{j}$ corresponde a la submatriz de rotación de la matriz de transformación homogénea entre los eslabones 4,5 y 6 , para lo cual

$$
\begin{aligned}
& \theta_{4}=-a \cos \\
& \left(-a_{z} \cdot \cos \left(\theta_{2}\right) \cdot \cos \left(\theta_{3}\right)+a_{z} \cdot \sin \left(\theta_{2}\right) \cdot \sin \left(\theta_{3}\right)\right) \\
& +\theta_{3}-11 \pi / 60
\end{aligned}
$$

$\theta_{5}=-a \sin \left(o_{x} \cdot \sin \left(\theta_{1}\right)-o_{y} \cdot \cos \left(\theta_{1}\right)\right)$

Para el planteamiento anterior, el número de posibles soluciones está limitada a los valores codo arriba y codo abajo de las coordenadas de nodo $\theta_{2}$ y $\theta_{3}$, mientras que las coordenadas $\theta_{1}$ dependerá del valor dado a la coordenada de la base deslizante, siendo imposible resolver el problema cinemático inverso sobre un conjunto cerrado y limitado de valores para las coordenadas nodales. La posibilidad de dar valor a priori a alguna de las coordenadas de nodo puede servir como una solución al problema de redundancia planteado. Pero, ¿cómo escoger el mejor valor a priori para una coordenada en particular? Dentro de las posibles soluciones a esta redundancia cinemática se encuentran los enfoques de descomposición de tareas [8]-[10], criterios de desempeño del manipulador para evitar singularidades y para evitar obstáculos [14],[15] y criterios basados en su dinámica [15],[16].

En este estudio se utilizan dos criterios, por una parte, se implementa el enfoque de descomposición de tareas, para lo cual se escoge de entre las posibles variables nodales la que permita una mayor flexibilidad para obtener de un lado la posición y por otro la orientación del elemento terminal. En el caso particular del robot en estudio, las coordenadas $d_{0}$ (desplazamiento sobre el riel) y $\theta_{1}$ (giro de la cintura del robot) son las que presentan esa característica. De esta manera, se proponen dos casos de estudio:

Caso $1 . \mathrm{d}_{0}$ asumido. Se toman diferentes valores $\mathrm{d}_{0}$ entre el mínimo $(0 \mathrm{~mm})$ y el máximo permitido por el riel $(1700 \mathrm{~mm})$. Con los diferentes valores $\mathrm{d}_{0}$, las expresiones en (4), (6) y (7) sirven para obtener conjuntos cerrados de soluciones para las coordenadas de giro $\theta_{1}, \theta_{2}$ y $\theta_{3}$.

Caso 2. $\theta_{1}$ asumido. Dando valores a $\theta_{1}$ entre los límites permitidos por el control que van desde $-135^{\circ}$ hasta $175^{\circ}$, se obtienen conjuntos solución para $\mathrm{d}_{0}, \theta_{2} \mathrm{y} \theta_{3}$. 
Conocido $\theta_{1}$, la coordenada de traslación se determina como,

$$
d_{0}=r_{y}-r_{x} \cdot \tan \left(\theta_{1}\right)
$$

En cualquiera de los dos casos, el número de valores que las variables $\mathrm{d}_{0}$ y $\theta_{1}$ pueden tomar son tantos como resolución tiene el control. De modo que se hace necesario determinar qué conjunto solución de todos los posibles es el más adecuado. La evaluación de ciertos índices de desempeño del manipulador para evitar singularidades en las diferentes configuraciones resultantes de la variación de las coordenadas articulares seleccionadas en los casos 1 y 2 servirá como criterio de selección de la solución final.

En la siguiente sección, se consideran algunos tipos de índices de desempeños que pueden usarse para determinar la cercanía a estados de singularidad.

\section{3. ÍNDICES DE DESEMPEÑO BASADOS EN LA DISTANCIA A LA SINGULARIDAD}

Los índices de desempeño son usados en los procesos de síntesis cinemática y dinámica (determinación de parámetros geométricos e inerciales) como parte del diseño de sistemas robóticos. Dentro de los principales índices se tienen aquellos que evalúan el desempeño cinetostático y que permiten evaluar la capacidad de transformar las velocidades y fuerzas de los actuadores de nodo o junta en velocidades y fuerzas del elemento terminal. La determinación de la gran mayoría de los índices de desempeño cinetostático están en función de la matriz jacobiana $J$, que representa las relaciones entre las velocidades del elemento terminal $\vec{v}$ con las velocidades de nodo $\overrightarrow{\dot{q}}$ y que dependen de la geometría y configuración del robot, a partir de la expresión,

$$
\vec{v}=J \times \overrightarrow{\dot{q}}
$$

Para el conjunto de todas las velocidades del elemento terminal realizables por las velocidades de junta, se satisface su norma Euclidiana $\|\vec{q}\| \leq 1$. Este conjunto de velocidades corresponde a un elipsoide girado en el espacio de $\vec{v}$. Así, sobre el eje mayor del elipsoide, el elemento terminal se puede mover a una mayor velocidad y, sobre su eje menor, lo hará a menor velocidad, pues estará más cerca a la singularidad, Fig. 6.
Fig. 6. ELIPSOIDE DE MANIPULABILIDAD

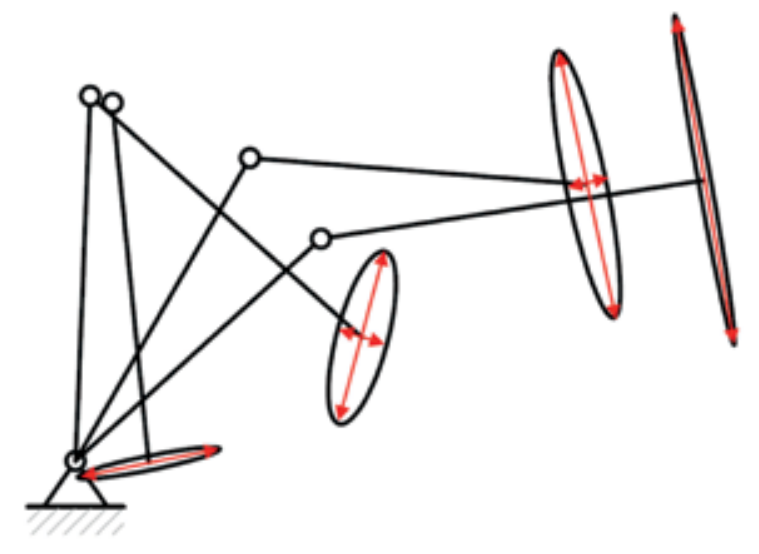

Fuente: los autores.

En algunos casos, la matriz jacobiana de un manipulador no es homogénea. Cuando se tienen manipuladores de posicionamiento y orientación, o cuando se tienen juntas prismáticas y de revolución, las unidades de los elementos de la matriz no son homogéneos. Desde el punto de vista numérico existen también diferencias en órdenes de magnitud que hacen que la matriz esté mal condicionada, de manera que los criterios usados pueden no representar una condición verdadera de funcionamiento del sistema. Para evitar estos problemas, la matriz jacobiana suele ser homogenizada o normalizada. En el primer caso, se suelen dividir los elementos asociados a las velocidades angulares por una longitud característica, existiendo diferentes propuestas para seleccionar dicha longitud [3], [11], [17]. En la normalización o equilibrado [18], la matriz jacobiana se obtiene

$$
J_{n}=E_{v} J E_{q}^{-1}
$$

donde $E_{v}=\operatorname{diag}\left(1 / v_{1_{\max }}, 1 / v_{2_{\max }}, \ldots, 1 / v_{n_{\max }}\right)$ y $\quad E_{q}=\operatorname{diag}\left(1 / \dot{q}_{1_{\max }}, 1 / \dot{q}_{2_{\max }}, \ldots, 1 / \dot{q}_{n_{\max }}\right)$ con $v_{i_{\max }}$ como la máxima velocidad angular o lineal de cada elemento $i$ de la velocidad del elemento terminal y $\dot{q}_{i_{\max }}$ como la máxima velocidad lineal o angular de la junta $i$.

A continuación se presentan diversos índices de desempeño que pueden ser usados para evaluar la cercanía a la singularidad en cada postura del robot.

\subsection{Número de condición}

El número de condición se obtiene como la relación entre el mayor y el menor valor singular de la matriz jacobiana. Para este propósito es nece- 
sario descomponer la matriz $J_{n}$ en valores singulares, de forma que

$$
J_{n}=U \sum V^{T}
$$

donde $\mathrm{U}$ y $\mathrm{V}$ son matrices ortogonales y $\Sigma$ contiene los valores singulares $\sigma_{i}$ de la matriz jacobiana. El número de condición para cada postura del manipulador se determina como,

$$
k=\frac{\sigma_{\max }}{\sigma_{\min }}
$$

Si el número de condición obtenido es cercano a la unidad, significa que la distribución de velocidades es uniforme y por lo tanto el manipulador puede moverse en cualquier dirección porque la distancia a la singularidad es grande. Si por el contrario, el número de condición es muy alto, el robot se acerca a una configuración singular.

\subsection{Mínimo valor singular}

Este índice de desempeño permite observar la proximidad de la configuración del robot a una disposición singular, de este modo muestra si la capacidad de algunos de los ejes principales del elipsoide (Fig. 6) está a punto de cancelarse. El valor de este índice va ser mayor cuando el volumen de velocidad del elipsoide sea mayor. Por lo que se busca tener un eje menor de la elipsoide suficientemente grande, lo que implica un mínimo valor singular lo más grande posible.

\section{3 Índice de manipulabilidad}

Lo que pretende el índice de manipulabilidad es medir la capacidad de generar velocidades en el elemento terminal en una cierta configuración. Además envuelve todos los casos generales incluyendo las configuraciones redundantes, de este modo queda definida como:

$$
w=\sqrt{\operatorname{det}\left(J_{n} \times J_{n}^{T}\right)}
$$

En este caso el producto de los valores singulares será el índice de manipulabilidad,

$$
w=\sigma_{1} \sigma_{2} \ldots \sigma_{m}
$$

Si la manipulabilidad es igual a cero significa que tendremos una configuración donde el robot pierde la capacidad de ejecutar movimientos en ciertas direcciones, en caso contrario, donde el valor de la manipulabilidad tienda a ser mayor, entonces tendremos una mayor capacidad de realizar movimientos y generar velocidades en el extremo final.

\section{RESULTADOS}

Con el fin de realizar las pruebas que permitan evaluar los diferentes índices de desempeño para el robot redundante, se seleccionan dos configuraciones específicas (dos puntos cualesquiera pertenecientes al espacio de trabajo del manipulador) y una trayectoria esférica de prueba, tal como se presenta en la Fig. 7.

\subsection{Solución para una única configuración}

Los puntos seleccionados para la prueba se obtienen de manera aleatoria dentro del espacio de trabajo del robot. En cada caso se obtiene la solución que mejor cumple con cada criterio de desempeño. En la tabla II se presentan los valores alcanzados para cada índice de desempeño.

TABLA II

VALORES DE ÍNDICES DE DESEMPEÑO EN LAS SOLUCIONES ALCANZADAS PARA PUNTOS PARTICULARES

\begin{tabular}{|c|c|c|c|c|}
\hline Punto & Casos & $\begin{array}{c}\text { Menor número de } \\
\text { condición }\end{array}$ & Máximo $\sigma_{\text {min }}$ & $\begin{array}{c}\text { Máximo índice de } \\
\text { manipulabilidad }\end{array}$ \\
\hline \multirow{2}{*}{1} & 1 & 849044 & $3,76 \times 10^{-4}$ & 4506 \\
\cline { 2 - 5 } & 2 & 808192 & $3,91 \times 10^{-4}$ & 4729 \\
\hline 2 & 1 & 101277 & $6,66 \times 10^{-3}$ & 3131 \\
\cline { 2 - 5 } & 2 & 29227 & $1,09 \times 10^{-2}$ & 3125 \\
\hline
\end{tabular}

Fuente: los autores.

De la Tabla se aprecia que para el primer punto no hay variaciones significativas que den como alternativa de solución un caso particular. Para el segundo punto puede apreciarse una diferencia considerable, tanto en los valores de condicionamiento de las matrices jacobianas y el máximo valor singular mínimo para la segunda solución (caso 2). Sería de esperar que lo mismo ocurriera con el índice de manipulabilidad. Sin embargo, se observa que el índice no permite observar esa diferencia entre soluciones. 
Fig. 7. PUNTOS Y TRAYECTORIA DE PRUEBA

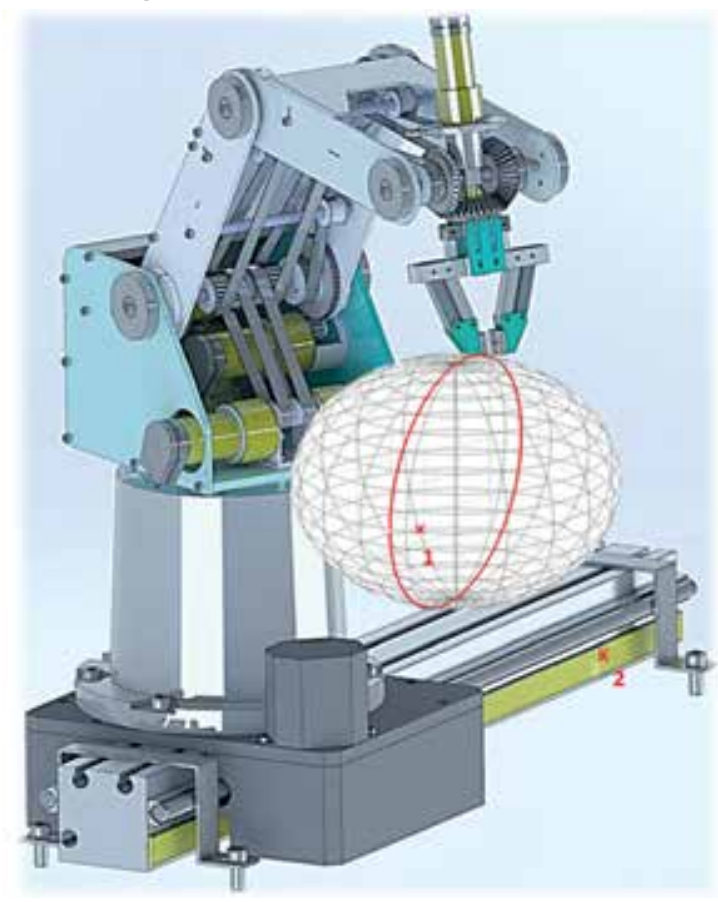

Fuente: Ios autores.

\subsection{Solución para una trayectoria}

Considerando la misma orientación utilizada en el ejemplo anterior, pero realizando el movimiento sobre la trayectoria esférica descrita, en la Tabla III se presentan los valores obtenidos en cada caso. Es de aclarar que para la trayectoria planificada se obtuvieron configuraciones singulares que fueron eliminadas de los datos de esta tabla con el fin de comparar los valores máximos y mínimos de cada criterio en cada caso. La trayectoria consta de 360 puntos.

De la Tabla puede observarse que el algoritmo de resolución de la cinemática inversa para el segundo caso, es decir, cuando se determina el valor de la coordenada de traslación $\mathrm{d}_{0}$ con cada variación del ángulo de la coordenada $\theta_{1}$ es computacionalmente de menor coste, medido en tiempo de ejecución. Tratándose de una trayectoria, lo más conveniente es determinar los valores que cada criterio alcanza en cada configuración. En la Fig. 8 se observa que para el Caso 2 se obtienen números de condición considerablemente menores, con lo cual estas soluciones están más alejadas de la singularidad comparadas con las soluciones dadas en el primer caso. Los altos condicionamientos obtenidos en ambos casos corresponden a las configuraciones singulares alcanzadas. En la Fig. 8, la abscisa denominada "Posición" hace referencia a cada punto de la trayectoria.

Fig. 8. VARIACIÓN DEL NÚMERO DE CONDICIÓN A LO LARGO DE LA TRAYECTORIA DE PRUEBA

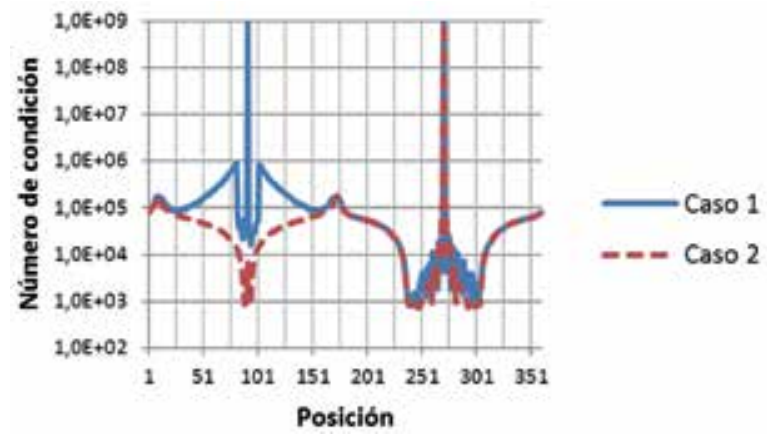

Fuente: los autores.

Para el criterio de maximizar el mínimo valor singular, Fig. 9, se observa que igual que en el criterio anterior, el Caso 2 permite obtener configuraciones más alejadas de la singularidad, dado que tienen valores singulares mínimos de mayor magnitud. Se observa también cómo la solución dada por el primer caso $\left(\mathrm{d}_{0}\right.$ variable) genera dos configuraciones singulares, mientras que el Caso 2 genera solamente una.

TABLA III

VALORES DE ÍNDICES DE DESEMPEÑO EN LAS SOLUCIONES ALCANZADAS PARA LA TRAYECTORIA

\begin{tabular}{|c|c|c|c|c|c|c|c|c|c|}
\hline \multirow{2}{*}{ Caso } & \multicolumn{2}{|c|}{ Menor número de condición } & \multicolumn{3}{c|}{ Máximo omin } & \multicolumn{2}{c|}{ Máximo índice de manipulabilidad } \\
\cline { 2 - 10 } & $\min$ & $\max$ & Tiempo, $s$ & $\min$ & $\max$ & Tiempo, $s$ & $\min$ & $\max$ & Tiempo, s \\
\hline 1 & 732 & $9,28 \times 10^{-5}$ & $1,79 \times 10^{-3}$ & $2,51 \times 10^{-4}$ & 0,343 & $1,64 \times 10^{-3}$ & 776 & 22743 & $1,66 \times 10^{-3}$ \\
\hline 2 & 715 & $1,72 \times 10^{-5}$ & $5,51 \times 10^{-4}$ & $2,34 \times 10^{-4}$ & 0,426 & $6,96 \times 10^{-4}$ & 672 & 31914 & $5,86 \times 10^{-4}$ \\
\hline
\end{tabular}

Fuente: los autores. 
Fig 9. VARIACIÓN DEL MÍNIMO VALOR SINGULAR A LO LARGO DE LA TRAYECTORIA DE PRUEBA

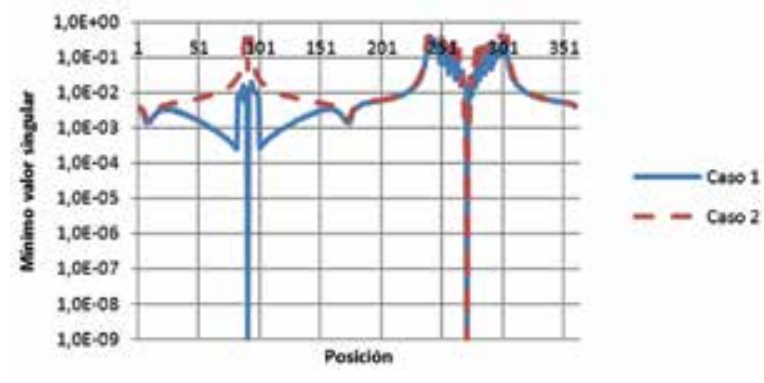

Fuente: Ios autores.

Se aprecia en las figuras anteriores que tanto el número de condición como el mínimo valor singular varían en orden de magnitud al comparar los casos de solución. Sin embargo, al analizar el criterio de índice de manipulabilidad, se observa de la Fig. 10, que aunque se nota claramente un mayor índice en el Caso 2, el orden de magnitud es comparativamente igual en ambos casos.

Fig. 10. VARIACIÓN DEL ÍNDICE DE MANIPULABILIDAD A LO LARGO DE LA TRAYECTORIA DE PRUEBA

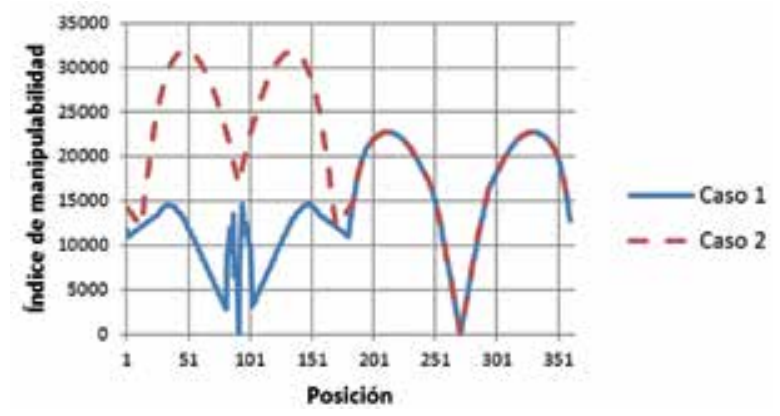

Fuente: los autores.

\section{CONCLUSIONES}

La utilización de índices de desempeño del manipulador, basados en su matriz jacobiana, pueden ser usados satisfactoriamente en la resolución del problema cinemático inverso de robots redundantes. Estas soluciones permiten seleccionar las configuraciones más alejadas de la singularidad con la ventaja adicional de ser de fácil programación y con tiempos de ejecución que permiten su implementación en tiempo real.

Tratándose de manipuladores redundantes, donde hay múltiples soluciones al problema cinemático inverso, es útil mediante un análisis previo determinar la preferencia de variable nodal a ser prefijada, tanto por mejorar el comportamiento cinemático global, basado en la distancia a la singularidad, como por buscar aquella que menos singularidades genera. Esta selección a priori de la variable prefijada está basada en el resultado promedio obtenido bajo una trayectoria. Para el caso particular del presente trabajo, cuando se prefija el ángulo $\theta_{1}$, se obtienen mejores índices de evaluación. Esta selección no genera valores óptimos locales, pero sí permite que al reducir el número de variables, se logre reducir considerablemente el tiempo de cálculo requerido.

Si bien en la literatura disponible, se mencionan los índices de valoración usados en este trabajo como criterios de resolución del problema cinemático inverso, no se conoce para cada tipo de manipulador cuál de ellos genera mejores resultados, pues estos índices dependen de la topología propia del manipulador. Para el manipulador estudiado, si bien con los tres criterios aplicados se obtuvieron resultados similares, el índice de manipulabilidad no es tan contundente al momento de comparar las soluciones, pues las diferencias entre los valores singulares máximos y mínimos pueden ser encubiertas al realizar el producto de todos los valores singulares.

En el presente trabajo se evaluó prefijar solamente dos variables nodales de acuerdo con la descomposición de tareas, sin embargo, para evitar soluciones correspondientes a configuraciones singulares, sería necesario introducir restricciones a los valores de los índices, de manera que por fuera de esos rangos se fijen otras coordenadas de nodo diferentes a las estudiadas.

\section{REFERENCIAS}

[1] S. G. Chiaverini, Oriolo, and I.D. Walker, "Kinematically Redundant Manipulators". Handbook of Robotics. Springer, 2008.

[2] T. Yoshikawa. Foundations of Robotics. Analysis and Control, The MIT Press, 1990.

[3] J. Angeles, Fundamentals of Robotic Mechanical Systems. Theory, Methods, and Algorithms, Springer, 1997.

[4] L. Huo and L. Baron, "Kinematic Inversion of Functionally-Redundant Serial Manipulators: Application to Arc-Welding", in Transactions- Canadian Society for Mechanical Engineering 29(4), 2005, pp. 679-690.

[5] L. Sciavicco and B. Siciliano, Modelling and Control of Robots Manipulator. Springer, 2000. 
[6] H. Adasa and J. Slotine, Robot Analysis and Control. John Wiley \& Sons, Inc. New York, 1992.

[7] J. J. Craig, Introduction to robotics. Mechanisms and control. Addison Wesley Publishing Company, 1989.

[8] F. Flacco and A. De Luca, "Fast Redundancy resolution for high- dimensional robots executing prioritized tasks under hard bounds in the joint space", in IEEE/ RSJ International Conference on Intelligent robots and systems, Tokyo, 2013.

[9] O. Kanoun, F. Lamiraux and P. F. Wieber, "Kinematic control of redundant manipulators: generalizing the task-priority framework to inequality task", in IEEE Transactions on Robotics, vol 27 (4), 2011.

[10] B. Siciliano, "Solving manipulator redundancy with the augmented task space method using the constraint Jacobian transpose", IEEE Int. Conf. on Robotics and Automation, vol. 5, pp. 1-8, 1992.

[11] J. Ángeles and F.C. Park, "Performance Evaluation and Design Criteria", Handbook of Robotics. Springer, 2008.

[12] C. Gosselin and J. Angeles, "A Global Performance Index for the Kinematic Optimization of Robotic Manipulators", Journal of Mechanical Design, 113(3), pp. 220-226, 1991.
[13] H. A. Moreno, R. Saltaren, I. Carrera, L. Puglisi and R. Aracil, "Índices de desempeño de robots manipuladores: una revisión del Estado del Arte", CEA Elsevier, España, 2012.

[14] J. Wang and C. M. Gosselin, "Kinematic Analysis and Design of Kinematically Redundant Parallel Mechanisms", Journal of Mechanical Design, vol. 126, pp. 109-118, 2004.

[15] J. Wu, J. Wang, L. Wang and T. Li, "Dynamics and control of a planar 3-DOF parallel manipulator with actuation redundancy". Mechanism and Machine Theory 44, pp. 835-849, 2009.

[16] S. B. Nokleby, R. Fisher, R. P. Podhorodeski and F. Firmani, "Force capabilities of redundantly-actuated parallel manipulators", Mechanism and Machine Theory 40, pp. 578-599, 2005.

[17] G. Cui, H. Zhang, F. Xu and C. Sun, "Kinematics Dexterity Analysis and Optimization of 4-UPS-UPU Parallel Robot Manipulator", Intelligent Robotics and Applications, vol. 8918, pp. 1-11, 2014.

[18] A. Harish and G. Satish Babu, "Manipulability Index of a Parallel Robot Manipulator", International Journal of Mechanical Engineering and Technology, vol. 6, Issue 6, pp. 09-17, 2015. 